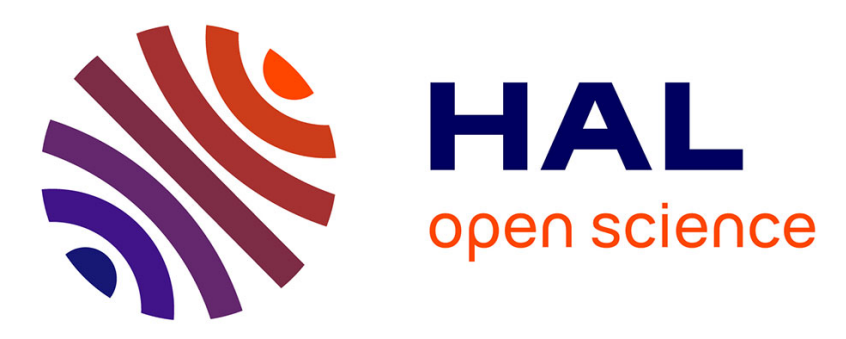

\title{
Identification of Fuzzy Relational Models for Fault Detection
}

Patrick Amann, Jean-Marc Perronne, Gérard Gissinger, Paul Martin Frank

\section{To cite this version:}

Patrick Amann, Jean-Marc Perronne, Gérard Gissinger, Paul Martin Frank. Identification of Fuzzy Relational Models for Fault Detection. Control Engineering Practice, 2001, 9, pp.555-562. 10.1016/S0967-0661(01)00016-8 . hal-00954560

\section{HAL Id: hal-00954560 https://hal.science/hal-00954560}

Submitted on 3 Mar 2014

HAL is a multi-disciplinary open access archive for the deposit and dissemination of scientific research documents, whether they are published or not. The documents may come from teaching and research institutions in France or abroad, or from public or private research centers.
L'archive ouverte pluridisciplinaire HAL, est destinée au dépôt et à la diffusion de documents scientifiques de niveau recherche, publiés ou non, émanant des établissements d'enseignement et de recherche français ou étrangers, des laboratoires publics ou privés. 


\title{
Identification of fuzzy relational models for fault detection ${ }^{\text {is }}$
}

\author{
P. Amann ${ }^{\mathrm{a}, \mathrm{b}}$, J.M. Perronne ${ }^{\mathrm{a}}$, G.L. Gissinger ${ }^{\mathrm{a}}$, P.M. Frank ${ }^{\mathrm{b}, *}$ \\ ${ }^{a}$ ESSAIM, Laboratoire MIAM, Université de Haute Alsace, 12, rue des Frères Lumière, F-68093 Mulhouse Cedex, France \\ ${ }^{\mathrm{b}}$ Fachgebiet Meß- und Regelungstechnik, Gerhard-Mercator-Universität-GH Duisburg, Bismarckstraße 81 BB, D-47048 Duisburg, Germany
}

Received 6 October 1999; accepted 11 August 2000

\begin{abstract}
This paper presents the concept of fuzzy relational models for use in a fuzzy output estimator. A suitable field of application is in fault diagnosis, where output observation rather than state observation is needed for the generation of fault reflecting residual signals. Due to their non-linear structure, fuzzy relational models can be used appropriately for building models of non-linear dynamic systems. In this paper, the identification of fuzzy models for residual generation is discussed. Emphasis is placed upon the model-building procedure including the identification of the model structure and of the parameters. As an application example, a real technical system is considered. The case study presents the detection of oversteering of a passenger car. The results of the application to residual generation are discussed. (C) 2001 Elsevier Science Ltd. All rights reserved.
\end{abstract}

Keywords: Fuzzy relational model; Fuzzy output observer; Model identification; Fault detection; Residual generation

\section{Introduction}

As mentioned by Frank (1996), there are several different approaches to fault detection. One of the most powerful is the model-based method, where a model is used to estimate the nominal output signals, appearing under error-free process conditions. This approach allows one to define the residuals as the difference between measurements and estimations. For this purpose, an output observer or an output estimator is required. In fact, the mismatch between measured and estimated output signals is considered to be caused by the effects of faults that have occurred in the process. This assumption is not exact, because there are always disturbances, e.g. due to measurement noise and modelling uncertainties. For effective fault detection, the influence of such disturbances on the residuals must be small in comparison with the effects of the faults.

The estimation of the error-free process outputs requires a model that describes the dynamic behaviour of

\footnotetext{
An early and shorter version of this paper by the authors, entitled "Identification of fuzzy relational models for fault detection" was presented at the 1999 IFAC World Congress in Beijing, P.R. China, July 1999.

* Corresponding author. Tel.: + 49-203-379-3387; fax: + 49-203379-2928.

E-mail addresses: g.gissinger@univ-mulhouse.fr (G.L. Gissinger), p.m.frank@uni-duisburg.de (P.M. Frank).
}

the process. Here, a distinction may be made between different kinds of models. The model can be given analytically by differential equations or qualitatively in the form of linguistic rules or in the form of a neural network-based or fuzzy-based parametric model whose parameters are identified through a set of measured input-output data.

The use of the first approach will be preferred, if a mathematical model of the system is available. Otherwise, if knowledge about the process is quite poor, one of the other methods should be applied. Either way, the dynamic behaviour of the system has to be described by linguistic rules that are based on the knowledge of human experts, or a set of measured data must be used to determine a data-based model in input-output description.

In this paper, a possible way of identifying a parametric model is presented. The model is based on fuzzy relational equations and is used to set up a fuzzy output estimator for residual generation.

\section{Model-building}

\subsection{Structure of fuzzy relational models}

The relational matrix is the kernel of a fuzzy relational model. This matrix describes the relationship between 


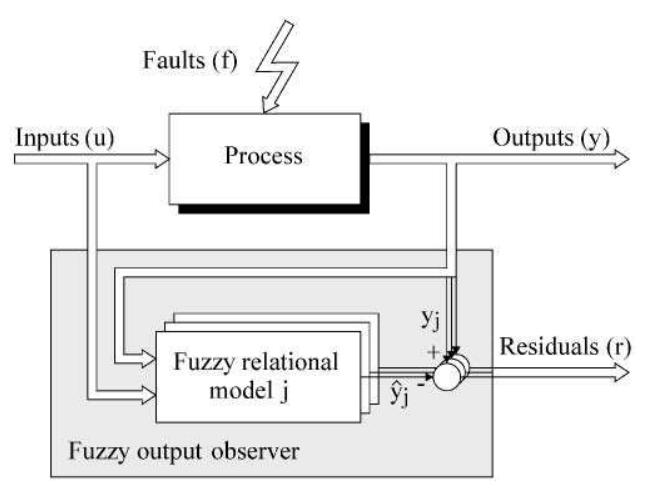

Fig. 1. Application of the fuzzy output observer to residual generation.

input and output variables (see Fig. 4). Its elements are the degrees to which the rules describing the dynamic behaviour of the system are possible. These parameters have to be identified according to the nominal process behaviour, reflected in the measured input-output data set. This stage provides the identification of the model and must be done with a set of measurements, which define the complete nominal behaviour under error-free process conditions. Otherwise, faults cannot be detected or fault alarms may arise, respectively.

The model is used in the second stage as an output estimator for the one-step-ahead prediction of the nominal output values and for the generation of the residuals. Details concerning the internal functionality of the relational model can be found in the paper from Amann, Perronne, Gissinger, and Frank (1999). If the process under consideration has more than one output, several relational models are needed, each of them dedicated to the estimation of one output signal. The resulting structure is depicted in Fig. 1.

As the fuzzy relational model is a black-box model, the task of model-building does not consist in finding "correct" physical parameters, whose values can be verified, but deals with matching a model to the measured inputoutput data. Hence, the best fitting model (matching to the complete nominal process behaviour) with the simplest structure and the smallest number of parameters must be found. It is evident, that several convenient models might exist. The selection criteria for the model to be used are complex and depend clearly on the application. In general, a human expert has to be consulted for this task. Special attention must be paid to the risk of choosing a local minimum. Although the model may achieve a very small estimation error in the case of the identification data, its performance might not be satisfactory during the evaluation stage with a different data set. In this case, the model is not able to predict the system behaviour correctly.

The core problem in fuzzy relational model-building is the search for the best-matching relational matrix. The result of this search depends on several parameters:
- The external data processing (sampling rate, filtering algorithms).

- The linguistic variables of all signals which are influencing the respective output signal (number, shape, position of the fuzzy sets).

- The structure of the model (input time delays, transfer orders).

- The identification data set (relevance and completeness of the measured data).

Thus, the complete procedure of system identification may be separated into three main tasks that will be discussed in the following: In the first step, the predefinitions to be given comprise the choice of sampling rate as well as the definition of the linguistic variables. In the second step, the structures of the submodels must be determined, which means that their input delays and orders must be chosen. In the third step, the parameters of the fuzzy relations have to be identified by the minimisation of an error criterion. In this procedure, the second step cannot be separated from the third one, because only the estimation error that has been achieved during model validation can be considered as a criterion for the performance of the respective model structure. Unfortunately, this estimation error depends on both previous tasks, so that the search for an optimised model is an iterative procedure.

\subsection{A-priori knowledge and predefinitions}

The design of a fuzzy relational model requires the availability of a-priori knowledge. As the model shall describe the input-output behaviour of the system in discrete time, the first definition to be given consists of determining the measurement filters. This includes the choice of the sample time as well as the design of any filtering algorithm for noise reduction or smoothing.

Afterwards, the linguistic variables have to be defined properly, which means that the universes of discourse have to be determined as well as the number and shapes of the linguistic terms. Here, the application of a clustering algorithm may be useful, such as, for example, the fuzzy clustering algorithm proposed by Kroll (1997).

\subsection{Structure identification}

The second stage of model-building consists of the choice of the model structure. For this purpose, a-priori knowledge from a human expert may be used. Otherwise, a structure identification algorithm as described in the following must be applied.

The task of structure identification concerns the definition of the multiple input-single output (MISO) submodels, that are needed to describe the behaviour of all process output signals. For each of these submodels, the 
relevant input signals have to be determined. Furthermore, it is necessary to know which samples of each signal have a direct influence on the actual output value. In fact, this information is related to knowledge of the input delays and the orders of the (non-linear) functions defining the system's behaviour. The knowledge of all required samples implies that delay and order are known, but not vice versa. However, the information about the input delays and the transfer order gives an idea of the relevant samples.

If no knowledge is available, each submodel comprises all process input signals as inputs and one process output signal as output. Of course, all previous samples up to the maximum delayed term have to be used for the estimation of the actual output value in this case. This leads to oversized fuzzy models and, thus, causes problems during the parameter identification.

- The choice of the input signals influencing a certain output, can be done by observation of the behaviour of the output signal relative to the changes of the inputs. This can give at least an idea of which input signals influence the respective output.

- The input delays can be determined by observation of the time delay occurring between a change in the input signal and the related reaction of the output. However, the exact measurement of the delay time may be difficult, especially in the presence of noise.

- The function orders can be approximated through the design of linear auto-regressive (ARX) models (Isermann, 1988). Here, the assumption must be made that the optimal order of the linear ARX models is also the optimum for the non-linear fuzzy relational model. This may hold if the ARX model achieves a good approximation of the system. Therefore, the validation of the ARX model is indispensable. Another possible way of determining the order of the system is given by computing the condition number of covariance matrices as proposed by Ljung (1987) and Isermann (1988).

If the methods mentioned above are applicable, the input delay and the order are known, so that only relevant samples have to be chosen in the last step. This can be done easily by checking all samples against their influence on the output signal. However, the results are not satisfactory sometimes. In this case, another systematic method must be applied:

- The easiest way to determine the sample points that are needed for the computation of the one step ahead prediction is to compare the estimation error achieved by all possible models. In this case, the maximum number of samples per variable $\kappa$ is increased starting from $\kappa=1$. For each $\kappa$, all possible combinations are evaluated and $\kappa$ is increased until the minimal estimation error $\varepsilon_{\min }(\kappa)$ increases. Here, the big variety of different models may lead to problems.
- This variety can be decreased by restricting to the choice of certain types of model. For instance, the evaluation may be limited to specific models that are defined through the input delay and the transfer order. Of course, this approach leads to problems, if the system cannot be described by this type of function.

- Another method makes use of the analysis of a data gradient vector. Schultz and Hillenbrand (1997) have proposed a method for the determination of the relevant samples by examining the data gradient vector. This method can be extended in the case of noisy measurements, captured on real systems (Schultz \& Krebs, 1997), but it does not allow a comparison of the relevance of different signals.

A comparison between the results provided by these methods is necessary in order that a suitable model is chosen. If the system structure is unknown, the choice of the model's structure is not evident. All methods may lead to local minima, which means, that the obtained model structure is satisfactory for the identification data, but does not describe the system's behaviour correctly in other nominal operational modes. The risk of choosing such a local model is minimised by a model validation. At this time, an expert is needed for the choice of the best solution.

\subsection{Parameter identification}

After having defined the structure of the model, the model parameters must be determined. In this stage, one looks for the parameter values that best fit the inputoutput data that has been measured at the process. The principle of the identification procedure is shown in Fig. 2.

In general, the parameter identification task is done by minimisation of a cost criteria, as for example the squared error, or by maximisation of the likelihood function. In this work, the least squares estimator (LSE) has been chosen, because it best fits to the problem studied and because it is easy to implement. Indeed, the

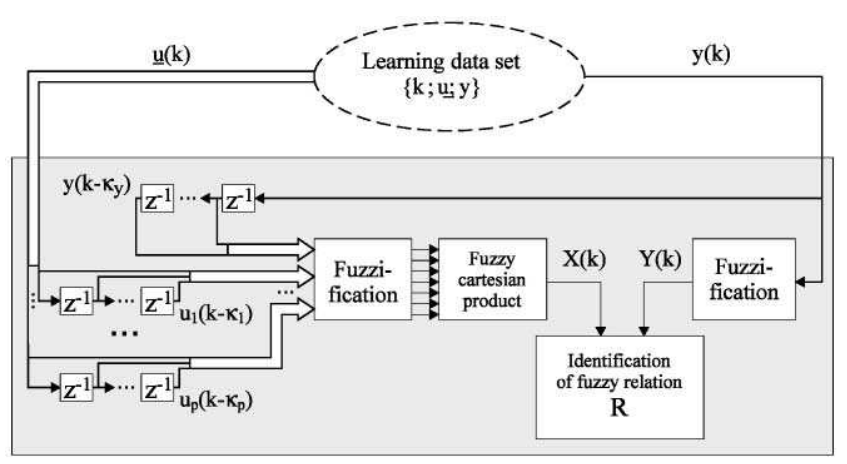

Fig. 2. Parameter identification structure of the fuzzy output observer. 
maximum likelihood criteria is equivalent to the least squares method, if the residuals are assumed to be Gaussian with zero mean values and known constant covariances (Ljung, 1987). The minimisation of the least squares criteria can be achieved by several different algorithms.

The method of Dubois (1992) allows the parameters for generalised norm and co-norm operators to be identified through an iterative search. It is based on the principle that the derivative of the (squared) error criterion $J$ equals zero for the optimal matrix:

$\frac{\partial J(R)}{\partial R}=0 \Rightarrow R=R_{\mathrm{opt}}$.

The use of generalised norm operators leads in general to robust models, but unfortunately, this iteration algorithm provides only a slow convergence.

The second method is the one that has been proposed by Jang, Sun, and Mizutani (1997) in the framework of adaptive neuro-fuzzy inference systems (ANFIS). It is based on a recursive LSE with efficient matrix inversion, as described by Ljung (1987). Here, the use of product and (bounded) sum as norm and co-norm operators is required. These operators lead to the most exact, but not to the most robust models. The identification algorithm is an iterative search for the best fitting parameters as well, but the convergence is much faster than with the one from Dubois'. The algorithm proceeds as follows:

$S_{i+1}=S_{i}-\frac{S_{i} X_{i+1} X_{i+1}^{\mathrm{T}} S_{i}}{1+\left[X_{i+1}^{\mathrm{T}} S_{i} X_{i+1}\right]}$,

$R_{i+1}=R_{i}+\left\langle S_{i+1} X_{i+1}\left[Y_{i+1}^{\mathrm{T}}-X_{i+1}^{\mathrm{T}} R_{i}\right]\right\rangle^{\mathrm{T}}$.

The initial values are $R_{0}=\mathbf{0}$ and $S_{0}=g \cdot \mathbf{I}$. For this method, the initialisation of the so-called covariance matrix $S$ plays an important role. A small value of $g$ leads to a very slow convergence. An increase of this initial value leads to a faster convergence, but, if the value of $g$ is too big, the algorithm skips the optimal parameters and does not reach the minimum error.

Another possibility is the use of gradient methods for the computation of optimal parameters (Isermann, 1988). These algorithms allow a recursive search for the parameters in the case of generalised operators. The first-order gradient method proceeds as follows:

$R(i+1)=R(i)-k \frac{\partial J(R)}{\partial R}$.

The influence of the step size $k$ on the estimation error is similar to that of the value $g$ on the recursive LS algorithm. In this work, a variable step size has been chosen, where $k$ is increased to $200 \%$ if the estimation error decreases during two following steps, otherwise it is decreased to $50 \%$. The first-order gradient method does not reach a very fast convergence.

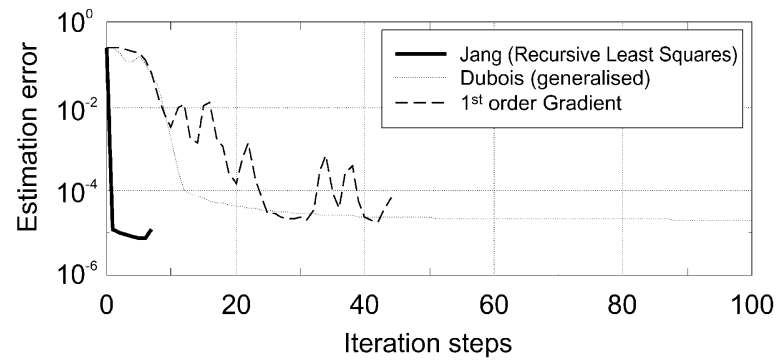

Fig. 3. Estimation error for different algorithms (vehicle subsystem $\alpha_{\mathrm{V}} \rightarrow \gamma_{\mathrm{t}}$ ).

The second-order gradient provides a faster convergence, but in this case, the inversion of the Hesse matrix may cause problems:

$R(i+1)=R(i)-\left[\frac{\partial^{2} J(R)}{\partial R^{2}}\right]^{-1} \frac{\partial J(R)}{\partial R}$.

This matrix will be ill conditioned or singular if not all of the modes of the system are excited by the measured input-output data. In this case, the second-order gradient algorithm cannot be applied.

The same problem arises for direct resolution. This method provides the direct (non-recursive) computation of the least squares estimate of the given input-output data and was described by Ljung (1987):

$R=\left\langle\left[\frac{1}{N} \sum_{k=1}^{N} X(k) X^{\mathrm{T}}(k)\right]^{-1} \frac{1}{N} \sum_{k=1}^{N} X(k) Y^{\mathrm{T}}(k)\right\rangle^{\mathrm{T}}$.

It requires the choice of product and bounded sum as norm and co-norm operators and the excitation of all modes of the system.

In general, each of the different methods has its advantages and its inconveniences. If a generalised norm operator is required, the algorithm Jang and the secondorder gradient method cannot be used. Fig. 3 shows the estimation errors for the vehicle subsystem $\alpha_{\mathrm{V}} \rightarrow \gamma_{\mathrm{t}}{ }^{1}$ Here, the covariance matrix has been initialised with $g=10$ and a variable step size has been chosen for the first-order gradient algorithm.

\section{Residual generations}

The fuzzy relational model whose set-up procedure is described in the previous section is used on-line for the residual generation at real or simulated processes. The structure of the fuzzy output estimator is depicted in Figs. 1 and 4.

In order to reinforce the effects of faults appearing in the process, a gain factor $\gamma$ is introduced, which enables

\footnotetext{
${ }^{1}$ See application, Section 5.
} 


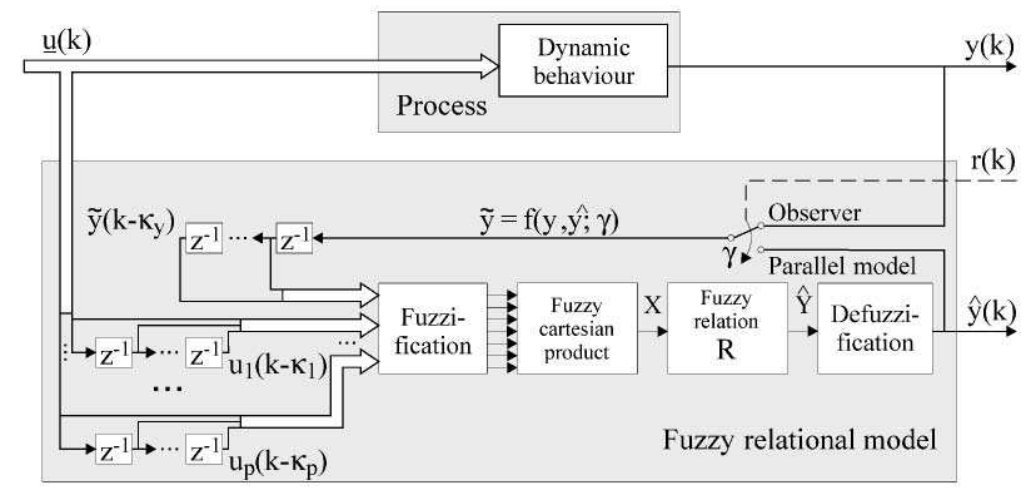

Fig. 4. Structure of fuzzy relational model.

switching between the operational modes "observer" (or "one-step-ahead predictor" more precisely) at $\gamma=0$ and "parallel model" at $\gamma=1$. During error-free process conditions $(r(k) \equiv 0)$, the gain is set equal to 0 , so that the model runs in observer mode. After the occurrence of an error $\left(r\left(k_{0}\right) \neq 0\right)$, the gain will be increased, so that the model does not adapt immediately to the effect of the fault, because it now runs in the parallel model mode. In fact, the switch will not be set equal to 1 , but to a value between 0.8 and 0.9 . Otherwise, it is not possible to detect further faults.

As the magnitude of the peak in the residuals is used to decide whether a situation will be considered as "normal" or "erroneous", the residuals must be normalised. The normalisation of the residual values can be done as proposed for example by Querelle, Mary, Kiupel, and Frank (1996). For this purpose, the mean value and the standard deviation are taken into consideration. The normalisation is done according to the equation $r_{n}(k)=\left(r(k)-\mu_{\text {nom }}\right) / 3 \sigma_{\text {nom }}$, where $\mu_{\text {nom }}$ and $\sigma_{\text {nom }}$ are mean value and standard deviation of the nominal residual values. Here, only residual values that appear under error-free conditions should be used.

\section{Implementation}

The realisation of the residual generator-cell by fuzzy output estimation has been carried out in the framework of a European project. The goal of the respective project was the development of a software tool for fault diagnosis in industrial processes. The fuzzy cell has been implemented under $\mathrm{C}++$ and provides a general configurability to different systems. The complete algorithm of the fuzzy output observer is divided into the two main tasks of configuration and residual generation.

The configuration procedure offers the possibility of easily designing a fuzzy output-observer module for almost every technical process. Therefore, at least one data set, containing measured input-output data and defining the dynamic behaviour of the system completely, is required. Some additional knowledge about the internal submodels (input delays, model orders and relevant samples) is recommended, but it can be replaced by automatic determination of the best-fitting model structure. For the parameter identification stage, several different methods can be chosen, depending on the norm operators and on the excitation of the system.

The data-flow in the run-time part is shown in Fig. 5, where $s_{i}$ is the number of fuzzy sets of the linguistic variable $U_{i}$ and $\kappa_{i}$ is the maximum delayed sample of the respective variable. In this part, the algorithm uses a previously generated relational model to estimate the system's output values and to derive the residual signals. This algorithm includes the normalisation of the residual values as well as switching between the functional modes "observer" and "parallel model" (given for each submodel through the gain factors $\gamma_{j}$ ).

\section{Application example: the automotive vehicle}

\subsection{System description}

The system under consideration is an automotive vehicle, and more precisely, its lateral dynamics during cornering. The goal of this application is to detect the oversteering of the vehicle (erroneous vehicle behaviour) before the driving situation gets critical. This early detection of oversteering should enable a driving assistance algorithm to avoid the dangerous situation or at least to reduce the risks. The data used for model identification has been measured directly at the test vehicle of the MIAM laboratory (Modélisation et Identification en Automatique et Mécanique) of the University of Mulhouse (France).

Fig. 6 shows a simplified description of a driven car: the driver defines a desirable trajectory to be followed. Therefore, he takes into consideration the actual position of the car on the road as well as external restrictions 


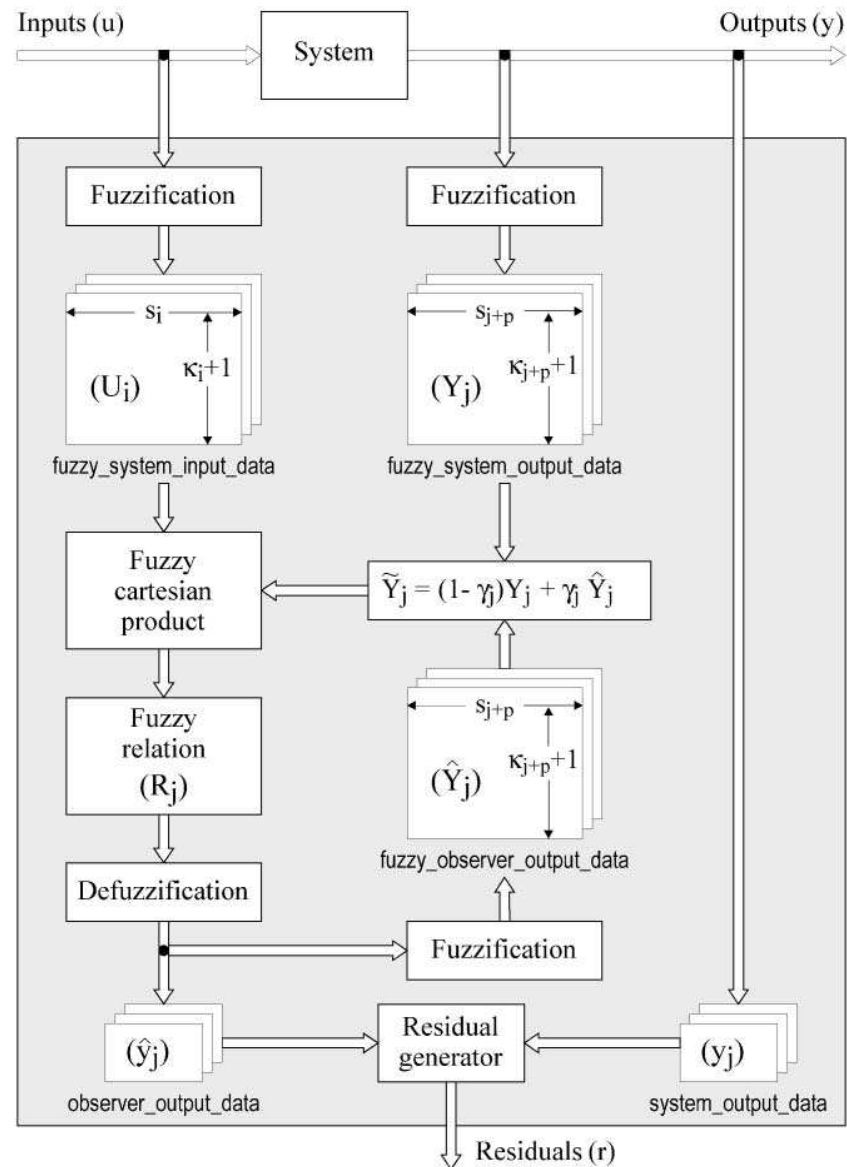

Fig. 5. Data flow in run-time algorithm.

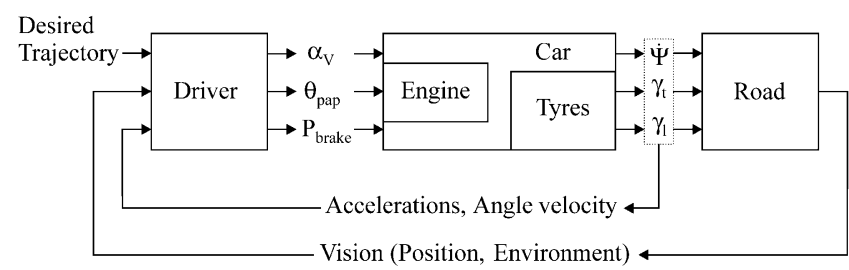

Fig. 6. Simplified system 'Driver-car-road'.

(traffic, obstacles etc.). Additionally, he is affected by the transversal acceleration $\gamma_{t}$, the longitudinal acceleration $\gamma_{1}$ and the yaw rate $\dot{\Psi}$. This perception enables him to manipulate the steering wheel angle $\alpha_{\mathrm{V}}$, to choose the engine order $\theta_{\text {pap }}$ and the braking power $P_{\text {brake }}$. The car (consisting of engine, tyres and other subsystems) reacts accordingly. Here, several non-linearities play an important role. Further details about the vehicle dynamics can be found in the books of Mitschke (1990) and Gillespie (1992).

To reproduce critical situations with a front-wheel driven vehicle, different experiments have been carried out. The different sensors and measurement parameters needed in the test car for these trials are shown in Fig. 7.

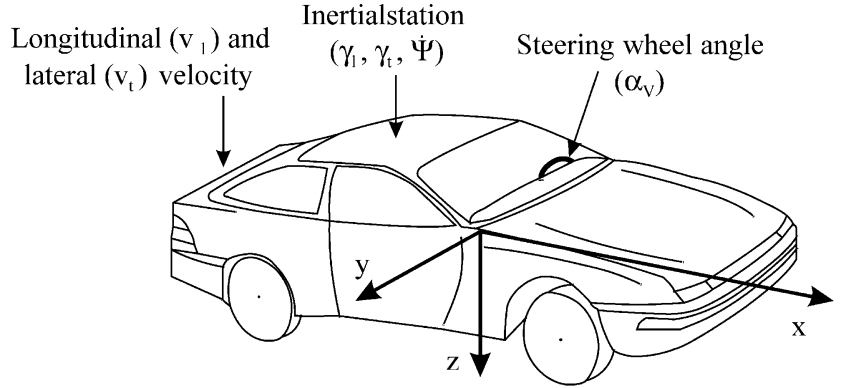

Fig. 7. Sensors and measurements in the test car.

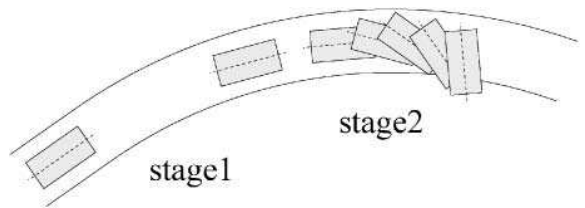

Fig. 8. Oversteering of a vehicle.

The longitudinal and lateral velocities are measured by an optical cross-correlation sensor located at the rear centre of the vehicle, whereas the gyro and acceleration sensors are located near the centre of gravity. All the signals are digitised by a data acquisition card inside a PC at the back of the test car.

While a vehicle is cornering regularly (stage 1 in Fig. 8), the steering wheel's angle causes a side-slip angle at the front tyres, which appears after a small time delay, due to the positioning of the tyres. Furthermore, a side-slip angle at the rear tyres is induced, which results in a sideslip of the centre of gravity, in the lateral acceleration of the car and in the yaw rate of the vehicle:

$\left.\begin{array}{r}\alpha_{\mathrm{V}} \rightarrow \delta_{\mathrm{f}} \\ \downarrow \\ \delta_{\mathrm{r}}\end{array}\right\} \Rightarrow\left\{\begin{array}{l}\delta_{\mathrm{C}} \rightarrow \gamma_{\mathrm{t}} \\ \dot{\Psi}\end{array}\right.$

If the driver exceeds the limits of stable cornering, the lateral acceleration $\gamma_{t}$ and the yaw rate $\dot{\Psi}$ increase, although the steering wheel angle $\alpha_{\mathrm{V}}$ remains constant: The back of the car breaks out and the car begins to oversteer (stage 2 in Fig. 8). As this situation is very dangerous, it should be avoided, because the driver risks losing control of his vehicle. The oversteering of the car can be provoked by suddenly taking the foot off the accelerator while cornering at the limit, or by cornering a curve too sharply relative to the actual speed (e.g. on a wet road).

\subsection{Model-building}

As mentioned above, oversteering of the vehicle will effect the time behaviour of the transversal acceleration $\gamma_{\mathrm{t}}$ and of the yaw rate $\dot{\Psi}$. Therefore, two models have 


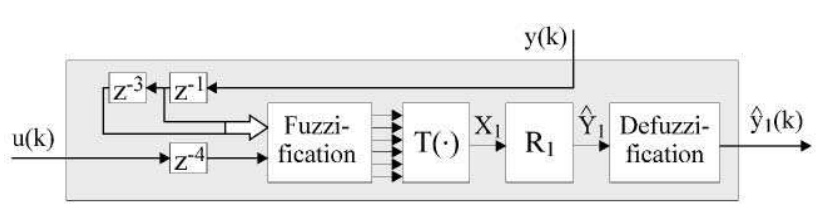

Fig. 9. Model 1: $\hat{Y}(k)=R_{1} \circ[U(k-4) \times Y(k-1) \times Y(k-4)]$.

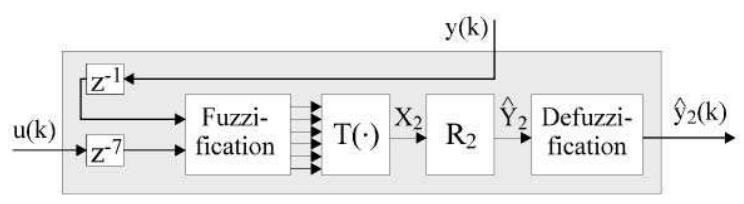

Fig. 10. Model 2: $\hat{Y}(k)=R_{2} \circ[U(k-7) \times Y(k-1)]$.

been designed, describing the relations $\alpha_{\mathrm{V}} \rightarrow \gamma_{\mathrm{t}}$ and $\alpha_{\mathrm{V}} \rightarrow \dot{\Psi}$, respectively. The residuals generated by the two observers are the normalised differences between measurement and estimation of $\gamma_{\mathrm{t}}$ and $\dot{\Psi}$. For the identification stage, two different measurement files have been used, reflecting the nominal behaviour of the car. Each of them contains two laps around the test track, sampled at $25 \mathrm{~Hz}$. The first file has been used for model identification, the second one for validation.

As an example, the model structures that have been determined by the different methods (see Section 2.3 above) for the subsystem $\alpha_{\mathrm{V}} \rightarrow \gamma_{\mathrm{t}}$ are compared in the following. The same procedure can be applied to the determination of the second subsystem $\alpha_{\mathrm{V}} \rightarrow \dot{\Psi}$ as well.

The application of different strategies for determining relevant samples leads to the following results:

- The complete check of all possible models leads in the present case to enormous computation times, so that the algorithm had to be stopped at order four. The minimum estimation error $\varepsilon=4.37 \times 10^{-5}$ has been obtained by a fourth-order model with an input delay of four samples. Its structure is depicted in Fig. 9.

- Using the second method with a restricted search algorithm, a model of first-order with an input delay of seven samples has been obtained. The resulting estimation error is $\varepsilon=4.12 \times 10^{-5}$. The model structure is shown in Fig. 10.

- The last method is based on the approach of Schultz and Hillenbrand (1997). In this case, the best model is of second-order and reaches an error of $\varepsilon=3.35 \times 10^{-3}$. Its structure is shown in Fig. 11 .

The choice of model 1 is based on a comparison between the models mentioned above, although the estimation error, obtained by model 2 is slightly smaller. Model 2 is only a first-order system with a big input delay of $280 \mathrm{~ms}$. A first-order system may describe one aspect of the car dynamics quite well, but will not be able to describe the complete behaviour of the system because it represents only a local minimum.

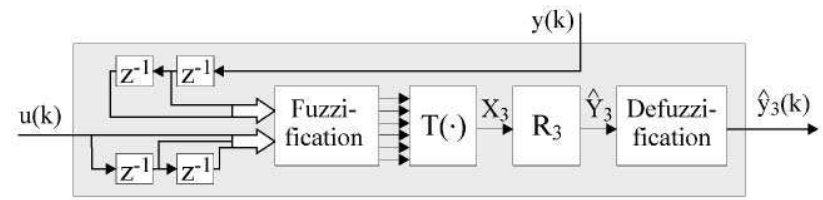

Fig. 11. Model 3: $\hat{Y}(k)=R_{3} \circ[U(k) \times U(k-1) \times U(k-2) \times Y(k-1) \times$ $Y(k-2)]$.
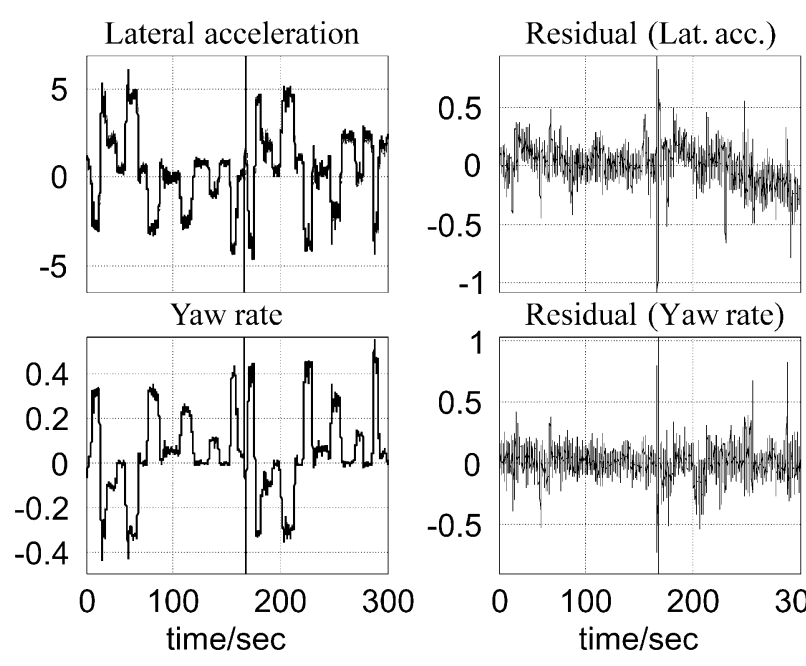

Fig. 12. Nominal residual behaviour.

\subsection{Simulation results}

The following results have been obtained with the models in Eq. (1). Figs. 12 and 13 depict the time behaviour of the steering wheel angle $\left(\alpha_{\mathrm{V}}\right.$ in $\left.{ }^{\circ}\right)$, of the lateral acceleration $\left(\gamma_{\mathrm{t}}\right.$ in $\left.\mathrm{m} / \mathrm{s}^{2}\right)$, of the yaw rate $\left(\dot{\Psi}\right.$ in $\left.{ }^{\circ} / \mathrm{s}\right)$ and of the normalised residual values.

$$
\begin{aligned}
\gamma_{\mathrm{t}}(k)= & f_{1}\left\{\alpha_{\mathrm{V}}(k-4) ; \gamma_{\mathrm{t}}(k-1), \gamma_{\mathrm{t}}(k-4)\right\}, \\
\dot{\Psi}(k)= & f_{2}\left\{\alpha_{\mathrm{V}}(k-2), \alpha_{\mathrm{V}}(k-3), \alpha_{\mathrm{V}}(k-4) ;\right. \\
& \dot{\Psi}(k-1), \dot{\Psi}(k-2)\} .
\end{aligned}
$$

Fig. 12 shows the residuals during nominal vehicle behaviour. The measured and estimated outputs are superimposed in the same plot. It reveals that the estimated outputs follow the measurements quite well, as the respective residuals remain in the nominal interval $[-1 ;+1]$.

In order to test the residual's behaviour in the case of oversteering, the fuzzy observers have been applied to a test file, where the driver oversteers his car. The results are shown in Fig. 13. The fault occurs in a left turn, so that steering wheel angle and yaw rate take negative values. The driver steers with an approximately constant angle between $t=42 \mathrm{~s}$ and $t=47.5 \mathrm{~s}$, but at $t=46.5 \mathrm{~s}$, the vehicle becomes unstable. This reveals by an increase of the yaw rate and of the lateral acceleration. At 


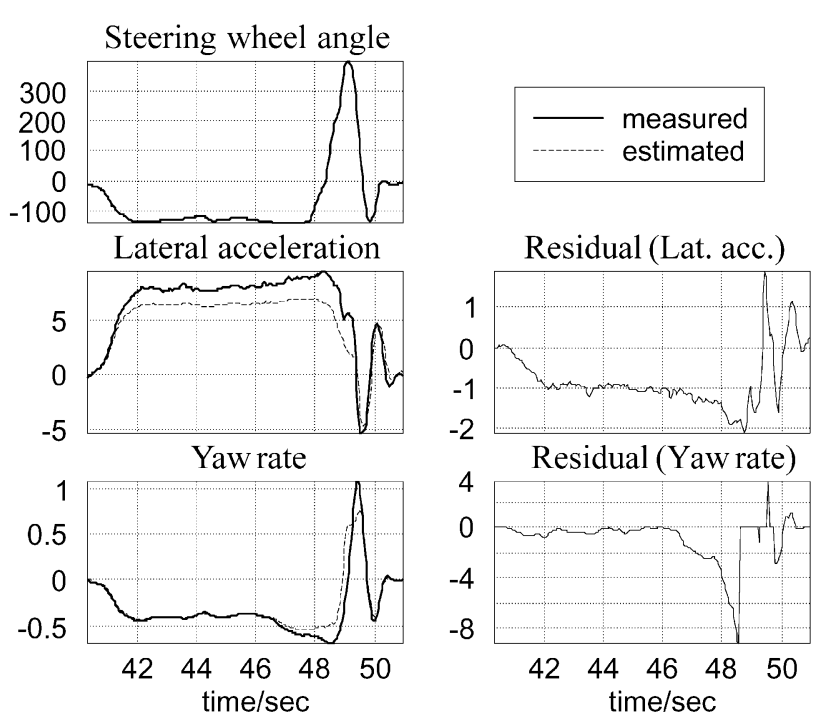

Fig. 13. Residuals in the presence of faults.

$t=47.5 \mathrm{~s}$, the driver tries to control the car by steering it in the opposite direction. The residuals of $\gamma_{t}$ and $\dot{\Psi}$ take both values greater than 1 , but it can be seen that the residual of $\dot{\Psi}$ behaves more reliably. It remains close to zero and clearly increases at $t=46.5 \mathrm{~s}$, whereas the residual of $\gamma_{t}$, already near to 1 , exceeds the limit very slowly and increases again after the reaction of the driver at $t=48 \mathrm{~s}$.

Thus, the residual of $\gamma_{t}$ should be considered very carefully in the early detection of oversteering. In contrast, the model $\alpha_{\mathrm{V}} \rightarrow \dot{\Psi}$ enables a clear detection of the "erroneous" behaviour of the vehicle.

\section{Conclusion}

In this paper, an approach for the design of fuzzy relational models and their application in fuzzy output estimators for fault detection has been presented. The principles and difficulties of model-building have been discussed. Finally, its application to a front-wheel driven vehicle has been investigated. The results obtained by the fuzzy output-estimators during the detection of oversteering show the ability of fuzzy relational models to the modelling of complex dynamic systems.

However, a human expert is still needed during the model-building stage, especially for the determination of the model structure. A completely automatic search algorithm risks ending up in a local minimum, thus providing a model that is not able to describe the system behaviour correctly. Here, additional knowledge must be assessed to decide which model should be chosen.

\section{References}

Amann, P., Perronne, J. M., Gissinger, G. L., \& Frank, P. M. (1999). Tuning fuzzy observers for residual generation. Proceedings of the European control conference, Karlsruhe, Germany, Paper F1053-1.

Dubois, G. (1992). Résolution d'un système d'équations de relations floues. Contribution à l'identification de systèmes complexes. Doctoral thesis, Université Nancy I, Nancy, France.

Frank, P. M. (1996). Analytical and qualitative model-based fault diagnosis - A survey and some new results. European Journal of Control, 2(1), 6-28.

Gillespie, T. D. (1992). Fundamentals of vehicle dynamics. Warrendale, PA: Society of Automotive Engineers.

Isermann, R. (1988). Identifikation Dynamischer Systeme, Band II. Berlin, Germany: Springer.

Jang, J. S. R., Sun, C. T., \& Mizutani, E. (1997). Neuro-fuzzy And Soft Computing. New York: Prentice Hall.

Kroll, A. (1997). Fuzzy-Systeme zur Modellierung und Regelung komplexer technischer Systeme. Fortschrittsbericht VDI Reihe $8 \mathrm{Nr} 612$, Düsseldorf, Germany: VDI-Verlag.

Ljung, L. (1987). System Identification: Theory for the User. New York: Prentice Hall.

Mitschke, M. (1990). Dynamik der Kraftfahrzeuge, Band C. 2. Aufl., Berlin, Germany: Springer.

Querelle, R., Mary, N., Kiupel, N., \& Frank, P. M. (1996). Use of qualitative modelling and fuzzy clustering for fault diagnosis. Proceedings of the WAC, Montpellier, France, vol. 5, pp. 527-532.

Schultz, J., \& Hillenbrand, S. (1997). Determining the structure of nonlinear models. Proceedings of the fourth European Control Conference, Bruxelles, Belgium, pp. 251-258.

Schultz, J., \& Krebs, V. (1997). Methoden der Strukturanalyse zur Identifikation nicht-linearer Prozesse. Proceedings of the 42nd International Wissenschaftl. Kolloquium, Ilmenau, Germany, pp. 125-132. 